\title{
Intensidade de pigmentação vermelha em maçãs e sua relação com os teores de compostos fenólicos e capacidade antioxidativa
}

\author{
Intensity of red pigmentation in apples and its influence on \\ phenolic compounds content and antioxidant activity \\ Danianni Marinho ZARDO ${ }^{1}$, Ana Paula DANTAS ${ }^{2}$, \\ Ricardo VANZ ${ }^{3}$, Gilvan WOSIACKI ${ }^{4}$, Alessandro NOGUEIRA ${ }^{5 *}$
}

\section{Resumo}

Os compostos fenólicos influenciam a qualidade de maçãs e seus produtos tanto em termos de aparência e sabor quanto em propriedades nutricionais. O objetivo deste trabalho foi quantificar os teores de compostos fenólicos totais, antocianinas, flavonóis e atividade antioxidante em maçãs da cultivar Gala e de duas mutações somáticas, Royal Gala e Galaxy Gala, com diferentes intensidades de pigmentação vermelha. Os teores de fenóis totais foram semelhantes nas frutas inteiras. A atividade antioxidante apresentou diferença significativa na fruta inteira, sendo que a cultivar Gala foi a que obteve maior valor $\left(17559 \mu \mathrm{m} \cdot \mathrm{g}^{-1}\right)$ e a cv. Galaxy Gala, com pouca pigmentação, o menor $\left(6160 \mu \mathrm{g} \cdot \mathrm{g}^{-1}\right)$. A cultivar Royal Gala apresentou valores intermediários de atividade antioxidante de $12050 \mu \mathrm{m} . \mathrm{g}^{-1}$, para as frutas com muita pigmentação vermelha, e $13546 \mu \mathrm{m} . \mathrm{g}^{-1}$ para as com pouca pigmentação. O teor de antocianinas na fruta inteira variou de 5,79 a $13,73 \mathrm{mg} .100 \mathrm{~g}^{-1}$ e o teor de flavonóis oscilou entre 23,37 e 382,83 mg.100 g-1 nas cvs. Royal Gala e Galaxy Gala, respectivamente. Analisado nas diferentes partes das maçãs, o epicarpo apresentou a maior concentração de fenóis com aproximadamente 57\% do conteúdo total e $42 \%$ da atividade antioxidante. Os compostos fenólicos e a atividade antioxidante nos sucos oxidados foram significativamente reduzidos com o escurecimento enzimático, com perdas de $69 \%$ e $83 \%$, respectivamente. As frutas de mesma cultivar com maior e menor intensidade de cor apresentaram teores de compostos fenólicos e capacidade antioxidante semelhantes, porém as cultivares variaram significativamente.

Palavras-chave: maçãs; coloração; suco de maçã; fenóis; atividade antioxidante.

\begin{abstract}
Phenolic compounds influence the quality of apples and their products in appearance, flavor, and nutritional properties. The aim of this work was to quantify the phenolic compounds, anthocyanins, flavonols, and antioxidant activity in Gala apples and of two somatic mutations, Royal Gala and Galaxy Gala, with different intensities of red pigmentation. The total phenolic content was similar in these fruits. The antioxidant activity presented significant difference in the whole fruits. gala variety presented the highest value $\left(17559 \mu \mathrm{m} . \mathrm{g}^{-1}\right)$ while Galaxy Gala, with low pigmentation, presented the lowest value $\left(6160 \mu \mathrm{m}_{\mathrm{g}} \mathrm{g}^{-1}\right)$. The anthocyanins content in the whole fruit varied from 5.79 to $13.73 \mathrm{mg} .100 \mathrm{~g}^{-1}$ and the flavonols content from 23.37 to $382.83 \mathrm{mg} .100 \mathrm{~g} \mathrm{~g}^{-1}$ in Royal Gala and Galaxy Gala, respectively. The phenolic compounds presented the highest concentration in the epicarp, around $57 \%$ of the total content, and $42 \%$ of the antioxidant activity. The phenolic compounds and the antioxidant activity in the apple juices were significantly reduced with the enzymatic browning, with losses of $69 \%$ and $83 \%$ respectively. The fruits from the same variety with high and low color intensity showed similar values of phenolic compounds content and antioxidant capacity. However, the cultivars varied significantly.
\end{abstract}

Keyworks: apple; color juice; apple juice; phenol; antioxidant activity.

\section{Introdução}

A maçã cultivar 'Gala', a mais plantada no Brasil compreendendo suas mutações somáticas, representa $46 \%$ da produção nacional, que é de 960.000 toneladas (ABPM, 2006). Essa cultivar apresenta elevada qualidade devido à sua suculência, ao sabor doce e à média acidez. Tem excelente aparência com uma coloração vermelho-rajada intensa e atraente no lado que recebe sol, embora o lado sombreado fique sem a coloração vermelha, destacando a cor de fundo amarelo-esverdeada (EPAGRI, 2002).

Entre as mutações somáticas da cultivar Gala que afetaram a cor do epicarpo, pode ser citada a Royal Gala e uma

Recebido para publicação em 16/8/2007

Aceito para publicação em 14/12/2007 (002758)

${ }^{1}$ Departamento de Engenharia de Alimentos, Universidade Estadual de Ponta Grossa - UEPG, Av. Carlos Cavalcanti 4748, Uvaranas, CEP 84030-900, Ponta Grossa - PR, Brasil

2 Grupo de Trabalho sobre Maçã, Departamento de Engenharia de Alimentos, Universidade Estadual de Ponta Grossa - UEPG, Av. Carlos Cavalcanti 4748, Uvaranas, CEP 84030-900, Ponta Grossa - PR, Brasil

3 Agrícola Fraiburgo, Rio das Pedras, CP 454, CEP 89560-000, Videira - SC, Brasil

4 Universidade Estadual de Ponta Grossa - UEPG, Av. Carlos Cavalcanti 4748, Uvaranas, CEP 84030-900, Ponta Grossa - PR, Brasil.

${ }_{5}^{5}$ Departamento de Engenharia de Alimentos, Universidade Estadual de Ponta Grossa, Av. Carlos Cavalcanti 4748, Uvaranas, CEP 84030-900, Ponta Grossa- PR, Brasil, E-mail: alessandro.nogueira@pesquisador.cnpq.br

${ }^{*}$ A quem a correspondência deve ser enviada 
de suas mutações, a Galaxy Gala. No primeiro caso, as frutas apresentam coloração mais intensa do que a Gala, com estrias mais pronunciadas em toda superfície, porém com menor intensidade no lado sombreado. No segundo, as frutas são ainda mais coloridas, apresentando estrias uniformemente distribuídas em toda a superfície do fruto, inclusive na parte sombreada. Na Europa, estas três cultivares são classificadas como (ABPM, 2007) vermelho-estriada e bicolor, (AWAD; DE JAGER; VAN WESTING, 2000) vermelho com estrias pronunciadas e (AWAD et al., 2001) vermelho-intenso e estriado uniforme, respectivamente (EPAGRI, 2002).

Esta pigmentação, relacionada à cultivar e à biossíntese de antocianinas em presença de luz, (SHAHIDI; NACZK, 2004) favorece a aparência atrativa das frutas, além de outros compostos fenólicos contribuírem de forma significativa com a sensação de amargor e adstringência (SHI; MAZZA; MAGUER, 2002) e na formação de aromas tanto na fruta quanto em seus produtos, com participação no flavor (LEA; DRILLEAU, 2003). Além desses aspectos tecnológicos, os fenóis têm um papel importante na dieta humana devido a sua ação antioxidante (PODSEDEK et al., 2000; OOMAH; MAZZA, 2000; SHAHIDI; HO, 2000; SHAHIDI; FINLEY, 2001).

Em maçãs, os compostos fenólicos estão localizados nos vacúolos (97\%), sendo que, nas células do epicarpo e subepicarpo, as suas concentrações são superiores àquelas encontradas nos tecidos internos da fruta (NICOLAS et al., 1994), e a sua composição pode ser característica de cada cultivar (PÉREZ-ILZARBE; HERNÁNDEZ; ESTRELLA, 1991; AWAD et al., 2001). Podem ser influenciados pelo grau de maturação, ambiente de plantio e condições de estocagem (SPANOS; WROLSTAD, 1992; LATTANZIO et al., 2001; VAN DER SLUIS et al., 2001) tanto qualitativa quanto quantitativamente, e estas diferenças apresentam grande interesse para aplicações tecnológicas e nutricionais (CAO et al., 1998; PODSEDEK et al., 2000; OOMAH; MAZZA, 2000; SHAHIDI; FINLEY, 2001).

A composição de compostos fenólicos em sucos de maçãs depende da cultivar utilizada para o processamento, das condições culturais e da forma de extração durante a prensagem, sendo possível também uma alteração de teores e de proporções como resultado de reações de escurecimento enzimático e formação de haze (SHAHIDI; NACZK, 2004).

A atividade antioxidante está relacionada à sua capacidade de liberação de hidrogênio que reage e elimina a ação negativa do oxigênio singlet (RICE-EVANS; MILLER; PAGANGA, 1997; KAUR; KAPOOR, 2001). No que concerne à saúde humana, os compostos fenólicos presentes nas plantas, incluindo as antocianinas, flavan-3-óis, procianidinas, flavonóis e ácidos fenólicos, são os responsáveis, juntamente com os carotenóides e a vitamina $\mathrm{C}$, pela diminuição do risco de várias doenças associadas com o estresse oxidativo (NOGUEIRA, 2003; LEA; DRILLEAU, 2003). Na maçã, a capacidade antioxidante é atribuída, principalmente, aos compostos fenólicos, como os flavonoides e os ácidos fenólicos (EBERHARDT; LEE; LIU, 2000; LEE et al., 2003) e não é influenciada pela vitamina C presente em sucos de maçãs (YANISHLIEVA-MASLAROVA; HEINONEN, 2001).
As antocianinas, um grupo de compostos fenólicos solúveis em água e de baixo potencial de oxi-redução (MATHEW; PARPIA, 1971; VAN DER SLUIS et al., 2000), responsáveis pela pigmentação de frutas, legumes, sucos e vinhos, apresentam combinações muito instáveis (GIL; HOLCROFT; KADER, 1997; ZABETAKIS; LECLERC; KAJDA, 2000; SHI; MAZZA; MAGUER, 2002), e seu conteúdo total pode ser afetado por fatores genéticos ou físicos, como luz e temperatura, podendo variar entre as frutas e em cultivares diferentes da mesma fruta (MAZZA; MINIATI, 1994). As mutações somáticas de algumas cultivares de maçã que produzem frutos uniformemente coloridos, mesmo aqueles sombreados, são de grande interesse comercial (CAMILO; DENARDI, 2002).

Os objetivos deste trabalho compreendem a avaliação dos compostos fenólicos totais, antocianinas, flavonóis e atividade antioxidante nas frutas e nos sucos de maçãs da cultivar Gala e de duas de suas mutações somáticas com diferentes intensidades de coloração vermelha.

\section{Material e métodos}

\subsection{Material}

Foram utilizadas três amostras de $18 \mathrm{~kg}$ das cultivares Gala, Royal Gala e Galaxy Gala, as duas primeiras produzidas na região de Fraiburgo (1048 m de altitude) e a terceira, na região de São Joaquim/SC (1350 m de altitude), fornecidas pela empresa Agrícola Fraiburgo. A cv. Gala com muita pigmentação vermelha foi utilizada como referência neste estudo e as cultivares Royal Gala e Galaxy Gala foram classificadas por sistema fotoelétrico de acordo com a pigmentação em dois grupos, (Figura 1): muito vermelha (MPV) e pouco vermelha (PPV).

\subsection{Métodos}

Inibição do escurecimento enzimático

Para impedir o escurecimento enzimático, foi utilizado cisteína. $\mathrm{HCl}$ monoclorohidratada na concentração de $2,0 \mathrm{mM} \cdot \mathrm{kg}^{-1}$ (SIGMA, C-7880).

\section{Preparo do material}

As maçãs foram analisadas inteiras e separadas em partes (epicarpo, mesocarpo e endocarpo), na presença do inibidor enzimático, e o procedimento de obtenção do extrato alcoólico para extração dos compostos fenólicos foi o mesmo. Após a amostragem, feita de forma aleatória com separação de $1 \mathrm{~kg}$ de frutas, estas passaram por um processo de seleção que englobou a retirada de maçãs com injúrias físicas ou com presença de patógenos e, na sequência, foram lavadas em água corrente e pesadas. De acordo com a Figura 2, as frutas inteiras foram trituradas em processador de alimentos (METVISA-MPA) e a massa ralada foi recolhida em béquer com inibidor do escurecimento enzimático (2,0 mM. $\left.\mathrm{kg}^{-1}\right)$, homogeneizada e colocada em contato com uma solução extratora composta de álcool 70: água: ácido fórmico 3\% (80:20:1) na proporção de 1:1 (p/v), conforme metodologia descrita por McGhie, Hunt e Barnett (2005) com 

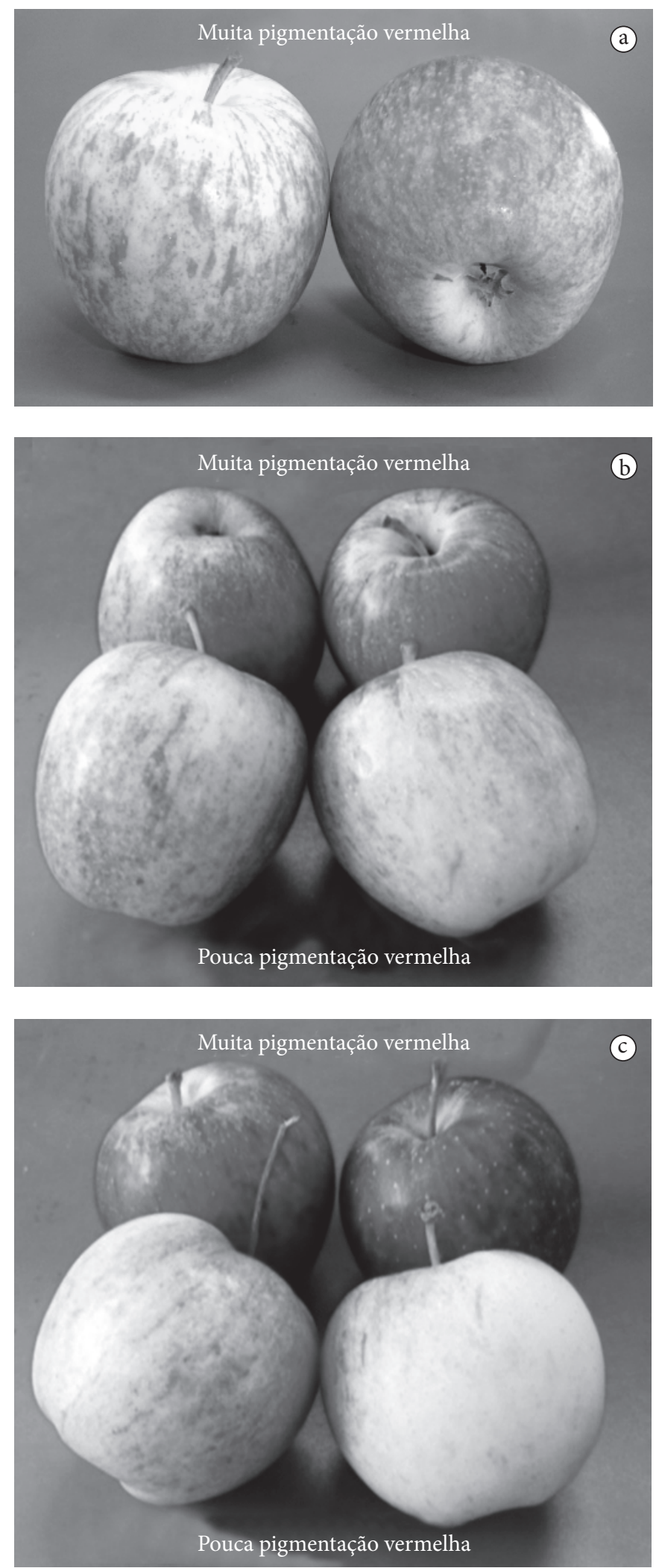

Figura 1. Amostras de maçãs a) cv. Gala; b) cv. Royal Gala; e c) cv. Galaxy Gala ilustrando a intensidade de pigmentação vermelha.

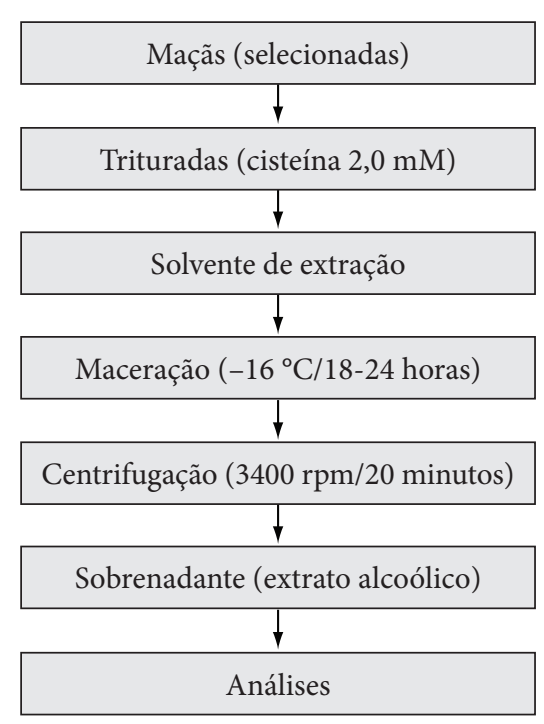

Figura 2. Fluxograma de obtenção dos extratos alcoólicos para análise das frutas (inteira e partes).

trituração em mixer vertical (Black \& Decker - SB40). Para as amostras que foram separadas em partes (mesocarpo, epicarpo e endocarpo), antes da trituração, as frutas foram descascadas de forma manual e o endocarpo (centro) foi retirado com um descaroçador ( $2 \mathrm{~cm}$ de diâmetro), restando o mesocarpo (polpa), em seguida, cada uma das três partes foi triturada. Todas as amostras foram mantidas a $-16^{\circ} \mathrm{C}$ durante 18 -24 horas e, em seguida, centrifugadas (CELM - COMBATE - Série 3548) por 20 minutos a $3400 \mathrm{rpm}$, e o sobrenadante (extrato alcoólico) foi separado para as análises.

\section{Processamento do suco}

Amostras de $5 \mathrm{~kg}$ das cultivares Gala, Royal Gala e Galaxy Gala, foram utilizadas neste processo. Os sucos foram processados na presença e na ausência do inibidor do escurecimento enzimático (cisteína.HCl monoclorohidratada). As maçãs foram divididas em dois grupos e, após seleção das frutas sadias, foram lavadas e trituradas. Durante o processo de trituração, em um grupo foi adicionado cisteína $\left(2,0 \mathrm{mM} \cdot \mathrm{kg}^{-1}\right.$ de fruta) e, no outro grupo, as frutas foram trituradas sem a adição de inibidor de escurecimento enzimático. Na sequência, a massa ralada foi prensada ( $3,0 \mathrm{kgf.cm}{ }^{2}$ durante 10 segundos), obtendose suco não-oxidado e oxidado, respectivamente. Em seguida, o suco foi despectinizado (Pectinex 3,0 mL.hL ${ }^{-1}$, Novozymes do Brasil) durante 60 minutos a $45^{\circ} \mathrm{C}$, trasfegado, clarificado (gelatina, 3,0 g.hL $\mathrm{hL}^{-1}$ e bentonite, 40,0 g.hL ${ }^{-1}$ ) e filtrado (papel filtro), dando origem ao suco clarificado. Por fim, os sucos foram pasteurizados (Figura 3).

\section{Determinação de compostos fenólicos totais}

Os fenóis totais foram determinados através de modificações da análise colorimétrica de Folin-Ciocalteau descrita por Singleton e Rossi (1965), tendo sido incluído um ensaio em 


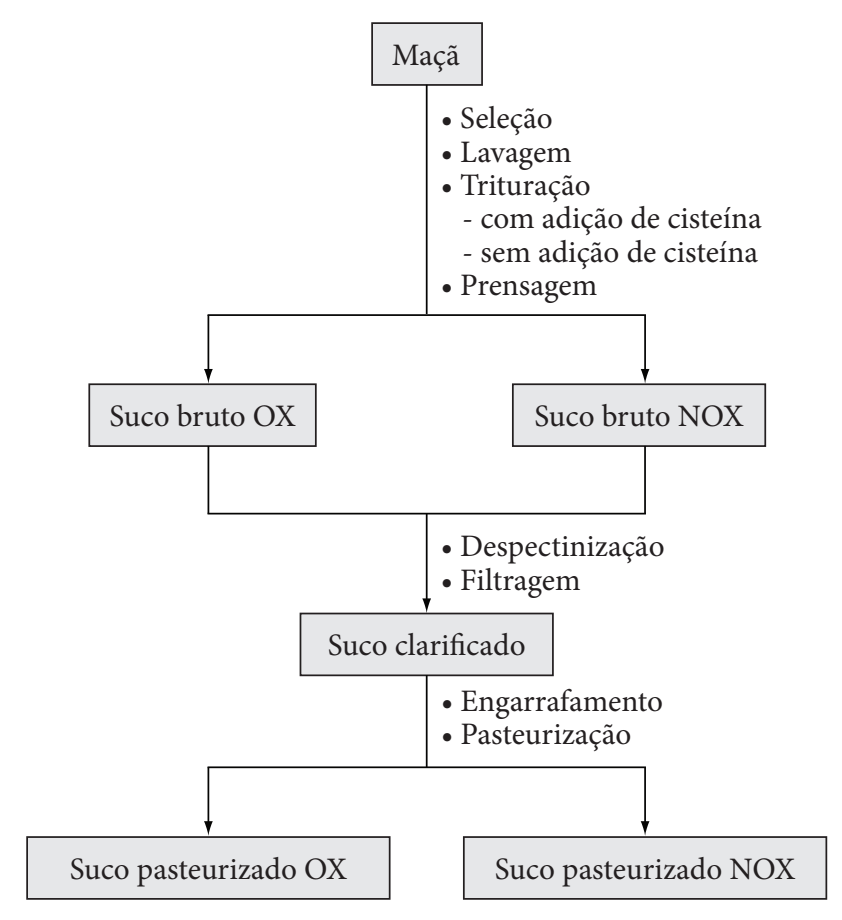

Figura 3. Fluxograma de obtenção de sucos de maçãs oxidado (OX) e não-oxidado (NOX).

branco para a solução líquido-extratora e outro para a solução de 2,0 mM de cisteína.HCl monoclorohidratada. As maçãs foram analisadas após o tempo de extração dos compostos fenólicos (18 a 24 horas), ou seja, no dia seguinte. Os resultados foram expressos em $\mu \mathrm{g} \cdot \mathrm{g}^{-1}$ de fenólicos totais, utilizando catequina (SIGMA, C-1251) como padrão $\left(200 \mu \mathrm{g} \cdot \mathrm{mL}^{-1}\right)$.

\section{Determinação da atividade antioxidante}

A atividade antioxidante foi determinada pela metodologia de FRAP, descrita por Benzie e Strain (1996) e Pulido, Bravo e Saura-Calixto (2000). As maçãs foram analisadas após o tempo de extração dos compostos fenólicos (18 a 24 horas), ou seja, no dia seguinte. A absorbância a $593 \mathrm{~nm}$ foi registrada usando o reagente FRAP como branco (L1). As leituras foram monitoradas durante 6 minutos (L6) a cada 15 segundos. A variação de absorbância entre L6 e L1 foi calculada para cada amostra, sendo traduzida em um valor de FRAP por proporcionalidade com a de uma solução de concentração conhecida - TROLOX $1000 \mu \mathrm{m}$ - testada em paralelo. O resultado foi expresso como $\mu \mathrm{m} . \mathrm{g}^{-1}$ de amostra. Um ensaio em branco foi feito para a solução líquido-extratora e para a do inibidor enzimático.

\section{Determinação de antocianinas e flavonóis}

O teor de antocianinas e flavonóis foi determinado pela metodologia descrita por Fuleki e Francis (1968). Após a extração das antocianinas e flavonóis com solvente extrator (etanol 95\% e $\mathrm{HCl} 1,5 \mathrm{~N}$, na proporção de 85:15 v/v), a absorbância a 535 e $374 \mathrm{~nm}$ foi medida, respectivamente. $\mathrm{O}$ total de antocianinas nas amostras foi expressa em mg. $100 \mathrm{~g}^{-1}$ de cianidina-3-galactosídeo, enquanto o teor de flavonóis foi expresso em mg.100 g-1 de quercetina, utilizando massa total da amostra, fator de diluição e o valor médio do coeficiente de absortividade molar para cada um dos compostos analisados.

\section{Análise estatística}

As análises foram realizadas em triplicatas e avaliadas mediante a análise de variância (ANOVA) e diferenciação das médias por teste de Tukey em nível de 5\% pelo programa Microsoft Office Excel 2003.

\section{Resultados e discussão}

\subsection{Compostos fenólicos totais}

Na Tabela 1, podem ser observados os resultados médios de compostos fenólicos totais para as cultivares Gala, Royal Gala e Galaxy Gala. Na fruta inteira, não foram observadas diferenças significativas no teor de fenóis totais entre as mutações somáticas, porém diferiram da cultivar Gala que apresentou o menor teor destes compostos $\left(906 \pm 22,49 \mu \mathrm{g} \mathrm{g}^{-1}\right)$. Os compostos fenólicos nas partes da fruta apresentaram diferenças significativas ao nível de 5,0\%, sendo que o epicarpo contribuiu com 49,0, 61,5 e 53,3\% dos fenóis da maçã inteira, o mesocarpo com 25,0, 16,2 e 17,3\% e o endocarpo com 26,0, 22,3 e 30,6\% para as cultivares Gala, Galaxy Gala e Royal Gala, respectivamente. Segundo Awad et al. (2000), as diferentes classes de fenóis não são sintetizadas e distribuídas uniformemente na maçã e são influenciadas, durante o desenvolvimento da fruta, por fatores externos (nutrientes disponíveis, temperatura e luminosidade).

No mesocarpo, as cultivares Gala e Royal Gala (PPV) apresentaram diferenças significativas frente às demais amostras analisadas no que diz respeito aos compostos fenólicos totais, apresentando os maiores teores. A cultivar Gala apresentou uma concentração de $227 \mu \mathrm{g} \cdot \mathrm{g}^{-1}$ de compostos fenólicos, enquanto a cultivar Royal Gala (PPV) apresentou um teor de $211 \mu \mathrm{g} . \mathrm{g}^{-1}$. Os teores de fenóis no endocarpo para a cultivar Royal Gala (MPV) e Galaxy Gala (PPV) não foram diferentes estatisticamente. $\mathrm{O}$ maior teor de compostos fenólicos para esta parte da maçã foi detectado na cultivar Royal Gala - PPV - $\left(407 \mu \mathrm{g} . \mathrm{g}^{-1}\right)$, o que representou $30,6 \%$ do total destes compostos na fruta (Tabela 1).

Com relação ao epicarpo, foi a parte da maçã com maior teor de compostos fenólicos, concordando com estudos de Nicolas et al. (1994), que demonstraram que na casca da maçã o teor de fenóis em relação à polpa pode ser de 3 a 10 vezes superior às outras partes da fruta. As cultivares Galaxy Gala (PPV) e Royal Gala (MPV) não apresentaram diferenças estatísticas nos teores de fenóis totais do epicarpo. De acordo com a Figura 1, pode-se observar que a cultivar Galaxy Gala (MPV), devido a sua intensa coloração vermelha, apresentou o epicarpo com o maior teor de fenóis totais $\left(745 \mu \mathrm{g} \cdot \mathrm{g}^{-1}\right)$, o que representa em torno de $61,5 \%$ do total dos fenóis para esta cultivar, contrapondo-se à cultivar Gala que apresentou um teor de $445 \mu \mathrm{g} \cdot \mathrm{g}^{-1}(49,0 \%)$.

\subsection{Atividade antioxidante}

Na Tabela 1, estão expressos os valores de atividade antioxidante. As análises na cultivar Gala, em todas as suas partes, 
apresentaram os maiores valores de atividade antioxidante, mesmo com baixo teor de fenóis. A cultivar Royal Gala (MPV e PPV) também merece atenção especial no que diz respeito à atividade antioxidante. Para esta cultivar, também foi obtido um elevado valor em todas as partes analisadas, embora o conteúdo de fenóis totais tenha se sobressaído somente no epicarpo, caracterizando assim um possível efeito varietal, o qual também foi observado por McGhie, Hunt e Barnett (2005), em cultivares de maçãs na Nova Zelândia.

No mesocarpo, a cultivar Gala foi a que obteve maior valor de atividade antioxidante $\left(4754 \mu \mathrm{m} . \mathrm{g}^{-1}\right)$, diferindo estatisticamente das demais amostras. As outras cultivares analisadas apresentaram pequenas diferenças, sendo que a cultivar Royal Gala (PPV) apresentou o menor valor para esta análise $\left(2148 \mu \mathrm{m} . \mathrm{g}^{-1}\right)$. Isso pode ser devido à presença de ácidos hidroxicinâmicos, como o ácido clorogênico, nesta parte da maçã; uma vez que, juntamente com os flavan-3-óis, como a catequina, também encontrada no mesocarpo da fruta, são importantes substratos para a polifenoloxidase (NICOLAS et al., 1994; OSZMIANSKI; LEE, 1990).

Ainda na Tabela 1, pode ser observado que o endocarpo das cultivares Royal Gala (PPV) e Gala possui um maior poder antioxidante (5182 e $5027 \mu \mathrm{m} . \mathrm{g}^{-1}$, respectivamente) comparado ao das cultivares Galaxy Gala (PPV e MPV) e Royal Gala (MPV), as quais foram significativamente diferentes entre si.

Com relação ao epicarpo das maçãs analisadas, a cultivar Gala apresentou um maior poder antioxidante comparado ao das outras cultivares analisadas. A cultivar Royal Gala, com pigmentação vermelha menos intensa que a Galaxy Gala (Figura 1), apresentou maior poder antioxidante devido aos elevados teores de flavonóis que foram $50 \%$ superiores (Tabela 2), o que reforça o baixo potencial de oxirredução das antocianinas (VAN DER SLUIS et al., 2000).

Com relação ao teor de flavonóis na fruta inteira, a cultivar Royal Gala (PPV) foi a que apresentou maior concentração $\left(382,83\right.$ mg. $\left.100 \mathrm{~g}^{-1}\right)$, de acordo com a Tabela 2. No epicarpo, a maior concentração deste grupo fenólico foi encontrada na culti- var Royal Gala (MPV). Este elevado teor de flavonóis encontrado na cultivar Royal Gala pode ser o responsável por sua atividade antioxidante ter sido superior a da cultivar Galaxy Gala.

O teor de antocianinas, tanto para a fruta inteira quanto para o epicarpo, foi maior nas cultivares com maior pigmentação vermelha, como era esperado (Tabela 2). O aumento de pigmentos antocianínicos na casca da maçã, atribuído a combinações de baixa temperatura durante a noite e elevados períodos de horas de sol durante o amadurecimento da fruta (REAY, 1999), podem ser, em parte, os responsáveis pelas diferenças na concentração de polifenóis na mesma cultivar.

Na Tabela 3, estão expressos os fatores de correlação e os valores de $\mathrm{R}^{2}$ entre os teores de compostos fenólicos e da atividade antioxidante nas cultivares Gala, Galaxy Gala e Royal Gala. Os valores de 0,63 a 0,99 indicam uma forte correlação entre os teores de fenóis e atividade antioxidante quando a fruta não é oxidada. A correlação, $\mathrm{R}^{2}=0,63$, na cultivar Galaxy Gala pode ser explicada pela variação de fenóis individuais na composição das cultivares; o que pode explicar a diferença encontrada no valor de atividade antioxidante (McGUIE; HUNT; BARNETT, 2005).

Tabela 2. Teores de antocianinas e flavonóis no epicarpo e maçã inteira das cultivares Galaxy Gala e Royal Gala em dois níveis de pigmentação vermelha.

\begin{tabular}{ccrc}
\hline Cultivar & \multicolumn{2}{c}{ Coloração } & \multicolumn{2}{c}{ Partes da fruta } \\
\cline { 2 - 4 } & \multicolumn{3}{c}{ Inteira } \\
& Antocianinas, mg.100 g $\mathrm{g}^{-1}$ \\
Galaxy Gala & MPV & $11,50 \pm 0,92$ & $537,11 \pm 2,36$ \\
& PPV & $5,79 \pm 0,25$ & $54,44 \pm 2,25$ \\
Royal Gala & MPV & $13,73 \pm 0,43$ & $327,97 \pm 6,07$ \\
& PPV & $6,09 \pm 1,55$ & $42,99 \pm 1,73$ \\
& Flavonol, mg.100 g-1 \\
Galaxy Gala & MPV & $60,69 \pm 1,30$ & $655,15 \pm 7,26$ \\
& PPV & $23,37 \pm 1,99$ & $558,57 \pm 2,74$ \\
Royal Gala & MPV & $38,57 \pm 0,84$ & $1163,39 \pm 11,20$ \\
& PPV & $382,83 \pm 1,56$ & $371,32 \pm 5,98$ \\
\hline
\end{tabular}

Tabela 1. Determinação de compostos fenólicos e da atividade antioxidante nas maçãs cultivares Galaxy Gala e Royal Gala, fruta inteira e suas partes, em dois níveis de pigmentação vermelha.

\begin{tabular}{|c|c|c|c|c|c|}
\hline \multirow[t]{2}{*}{ Cultivar } & \multirow[t]{2}{*}{ Coloração } & \multicolumn{4}{|c|}{ Partes da fruta } \\
\hline & & Inteira & Epicarpo & Mesocarpo & Endocarpo \\
\hline \multicolumn{6}{|c|}{ Fenóis totais $\left(\mu \mathrm{g} \cdot \mathrm{g}^{-1}\right)$} \\
\hline Gala & & $906^{b} \pm 22,49$ & $445^{\mathrm{d}} \pm 19,34$ & $227^{\mathrm{a}} \pm 7,36$ & $234^{\mathrm{bc}} \pm 5,48$ \\
\hline \multirow[t]{2}{*}{ Galaxy Gala } & MPV & $1146^{\mathrm{a}} \pm 47,58$ & $745^{a} \pm 19,34$ & $181^{\mathrm{b}} \pm 7,36$ & $219^{c} \pm 5,48$ \\
\hline & PPV & $1086^{a} \pm 22,49$ & $629^{\mathrm{b}} \pm 35,30$ & $181^{\mathrm{b}} \pm 9,72$ & $276^{\mathrm{b}} \pm 19,92$ \\
\hline \multirow[t]{2}{*}{ Royal Gala } & MPV & $1096^{\mathrm{a}} \pm 29,78$ & $636^{\mathrm{b}} \pm 12,71$ & $178^{\mathrm{b}} \pm 11,58$ & $282^{\mathrm{b}} \pm 9,01$ \\
\hline & PPV & $1149^{\mathrm{a}} \pm 24,68$ & $536^{c} \pm 15,43$ & $211^{\mathrm{a}} \pm 13,02$ & $407^{\mathrm{a}} \pm 23,87$ \\
\hline \multicolumn{6}{|c|}{ Atividade antioxidante $\left(\mu \mathrm{m} \cdot \mathrm{g}^{-1}\right)$} \\
\hline Gala & & $17559^{\mathrm{a}} \pm 22,49$ & $8111^{\mathrm{a}} \pm 19,34$ & $4754^{\mathrm{a}} \pm 7,36$ & $5027^{a} \pm 5,48$ \\
\hline \multirow[t]{2}{*}{ Galaxy Gala } & MPV & $6526^{\mathrm{d}} \pm 153,93$ & $3130^{c} \pm 645,43$ & $2353^{b c} \pm 156,30$ & $1047^{\mathrm{d}} \pm 152,73$ \\
\hline & PPV & $6160^{\mathrm{d}} \pm 329,04$ & $1530^{\mathrm{d}} \pm 361,56$ & $2148^{\mathrm{b}} \pm 84,59$ & $2471^{\mathrm{c}} \pm 88,05$ \\
\hline \multirow[t]{2}{*}{ Royal Gala } & MPV & $12050^{c} \pm 486,96$ & $6388^{\mathrm{b}} \pm 211,27$ & $2483^{\mathrm{b}} \pm 156,30$ & $3897^{\mathrm{b}} \pm 233,16$ \\
\hline & PPV & $13546^{\mathrm{b}} \pm 519,18$ & $5655^{\mathrm{b}} \pm 296,10$ & $2714^{\mathrm{b}} \pm 119,94$ & $5182^{\mathrm{a}} \pm 241,07$ \\
\hline
\end{tabular}

$\mathrm{MPV}=$ muita pigmentação vermelha; $\mathrm{e} P \mathrm{PV}=$ pouca pigmentação vermelha 
Os compostos fenólicos nos sucos oxidados (OX) foram drasticamente reduzidos com o escurecimento enzimático. Isto pode ser observado nas três cultivares analisadas; a Royal Gala com pouca pigmentação vermelha, apresentou teores de fenóis totais de 955,22 mg. $\mathrm{L}^{-1}$, após a oxidação enzimática, estes teores passaram a 166,17 mg. $\mathrm{L}^{-1}$, o que representa perdas na ordem de $82 \%$. Já na cultivar Galaxy Gala, com pouca pigmentação vermelha, os teores de compostos fenólicos passaram de 955,22 mg.L $\mathrm{L}^{-1}$ para 166,17 mg. $\mathrm{L}^{-1}$, com o processo oxidativo, e a cultivar Gala teve seus teores de fenóis reduzidos de 705 a 367 mg.L-1 (Tabela 4). Esta variação ocorre em função da composição fenólica, substrato da polifenoloxidase, encontrada em cada uma das cultivares, bem como da concentração e da atividade da enzima (SHAHIDI; NACZK, 2004; NOGUEIRA et al., 2003).

Os teores de fenóis totais para os sucos das amostras MPV e PPV sem oxidação foram semelhantes, porém estes valores, após a reação de escurecimento enzimático, foram diferentes. A oxidação enzimática afetou os teores de fenóis totais de forma mais intensa nas maçãs com pouca pigmentação vermelha, com redução em torno de 79\%, o que indica que as amostras MPV apresentaram um suco com maior teor de compostos fenólicos de baixa oxidação, possivelmente antocianinas (Tabela 4).

Os valores de atividade antioxidante nos sucos nãooxidados (NOX) foram superiores aos encontrados nas frutas in natura. Este fato pode ser proveniente do próprio processo de extração, pois determinadas classes de compostos fenólicos que apresentam elevada atividade antioxidante, como a cianidina 3-galactosídeo, procianidinas, quercetina, ácido clorogênico e floridzina, presentes nos vacúolos celulares, juntamente com

Tabela 3. Fatores de correlação entre os teores de compostos fenólicos e da atividade antioxidante das cultivares Gala, Galaxy Gala e Royal Gala e respectivos valores de $\mathrm{R}^{2}$.

\begin{tabular}{lccc}
\hline Cultivar & Coloração & Fator de correlação & $\mathrm{R}^{2}$ \\
\hline Gala & - & 19,348 & 0,99 \\
Galaxy Gala & $\mathrm{MPV}$ & 5,3695 & 0,82 \\
& $\mathrm{PPV}$ & 5,1764 & 0,56 \\
\multirow{4}{*}{ Royal Gala } & $\mathrm{MPV}$ & 11,049 & 0,98 \\
& $\mathrm{PPV}$ & 11,711 & 0,99 \\
\hline
\end{tabular}

outros compostos (açúcares e ácidos orgânicos) são facilmente extraídos durante o processamento (TSAO et al., 2005).

A reação de escurecimento enzimático diminuiu significativamente os valores de atividade antioxidante dos sucos de maçã. Essa perda variou entre 70 e $94 \%$ para a cv. Galaxy (MPV) e cv. Galaxy (PPV), respectivamente. Estas diminuições foram maiores para as cultivares com pouca pigmentação vermelha, o que pode estar relacionado com o baixo potencial de oxirredução das antocianinas (VAN DER SLUIS et al., 2000).

\section{Conclusões}

Amostras de maçãs provenientes da mesma região e de mutações somáticas da mesma cultivar variam muito entre si no que diz respeito aos teores de compostos fenólicos e atividade antioxidante. Frutas com muita pigmentação vermelha apresentaram atividade antioxidante semelhante às frutas da mesma cultivar com pouca pigmentação vermelha. O elevado teor de atividade antioxidante no epicarpo é principalmente devido aos teores de flavonóis. Os maiores teores de compostos fenólicos e uma elevada atividade antioxidante foram encontrados no epicarpo das frutas. A reação de escurecimento enzimático foi a principal responsável pelas perdas de fenóis e atividade antioxidante em sucos de maçãs.

\section{Agradecimentos}

Os autores agradecem à Universidade Estadual de Ponta Grossa por ceder os laboratórios para a realização deste trabalho, à Agrícola Fraiburgo/SC por ceder amostras de maçãs, bem como aos órgãos de fomento $\mathrm{CNPq}$, CAPES/PRODOC pelo fornecimento das bolsas que subsidiaram o trabalho dos pesquisadores, e ao Grupo de Trabalho sobre Maçã - GTM (www.uepg.br/gtm), pelo apoio complementar.

\section{Referências bibliográficas}

Associação Brasileira de Produtores de Maçã - ABPM. Dados estatísticos sobre a cultura da macieira. Disponível em: <http:://www.abpm.org.br>. Acesso em: 04 Fev. 2007.

Tabela 4. Determinação de compostos fenólicos e da atividade antioxidante nos sucos oxidados e não-oxidados das cultivares Galaxy Gala e Royal Gala em dois níveis de pigmentação vermelha.

\begin{tabular}{|c|c|c|c|}
\hline Cultivar & Cor do epicarpo & Fenóis totais mg. $\mathrm{L}^{-1}$ & Atividade antioxidante $\mu \mathrm{m} \cdot \mathrm{g}^{-1}$ \\
\hline \multirow[t]{4}{*}{ Galaxy Gala } & MPV- NOX & $813,27^{\mathrm{b}} \pm 33,38$ & $15805,95^{\mathrm{b}} \pm 616,77$ \\
\hline & MPV-OX & $423,88^{c} \pm 12,11$ & $4593,11^{c} \pm 44,44$ \\
\hline & PPV-NOX & $970,15^{a} \pm 26,02$ & $22793,43^{a} \pm 307,86$ \\
\hline & PPV-OX & $235,82^{\mathrm{d}} \pm 15,80$ & $1293,43^{d} \pm 20,33$ \\
\hline \multirow[t]{4}{*}{ Royal Gala } & MPV- NOX & $957,87^{\mathrm{a}} \pm 5,27$ & $16970,27^{\mathrm{a}} \pm 461,66$ \\
\hline & MPV-OX & $287,89^{\mathrm{b}} \pm 5,17$ & $3392,80^{b} \pm 75,31$ \\
\hline & PPV-NOX & $955,22^{\mathrm{a}} \pm 11,66$ & $16610,33^{\mathrm{a}} \pm 572,52$ \\
\hline & PPV-OX & $166,17^{\mathrm{c}} \pm 29,87$ & $1990,61^{\mathrm{c}} \pm 8,13$ \\
\hline \multirow[t]{2}{*}{ Gala } & NOX & $705^{\mathrm{a}} \pm 5,31$ & $14072^{\mathrm{a}} \pm 625,89$ \\
\hline & OX & $367^{b} \pm 20,86$ & $4008^{\mathrm{b}} \pm 88,28$ \\
\hline
\end{tabular}

MPV = muita pigmentação vermelha; $\mathrm{PPV}=$ pouca pigmentação vermelha; $\mathrm{OX}=$ oxidado; $\mathrm{e}$ NOX = não-oxidado. 
AWAD, M. A.; De JAGER, A.; Van WESTING, L. M. Flavonoid and clorogenic acid levels in apple fruit characterization of variation. Science Horticultural, v. 83, n. 3-4, p. 249-263, 2000.

AWAD, M. A. et al. Flavonoid and chlorogenic acid changes in skin of «Elstar» and «Jonagold» apples during development and ripening. Science Horticultural, v. 90, n. 1-2, p. 69-83, 2001.

BENZIE, I. F. F.; STRAIN, J. J. The ferric reducing ability of plasma (FRAP) as a measure of "antioxidant power": the FRAP assay. Analytical Biochemistry, v. 239, n. 1, p. 70-76, 1996.

CAMILO, A. P.; DENARDI, F. Cultivares: Descrição e comportamento no sul do Brasil. In: EPAGRI. A cultura da macieira. Florianópolis: EPAGRI, 2002. Cap. 5, p. 113-168.

$\mathrm{CAO}, \mathrm{G}$. et al. Increase in human plasma antioxidant capacity after consumption of controlled diets high in fruits and vegetables. American Journal of Clinical Nutrition, v. 68, p. 1081-1087, 1998.

EBERHARDT, M. V.; LEE, C. Y.; LIU, R. H. Antioxidant activity of fresh apples. Nature, v. 405, n. 6789, p. 903-904, 2000.

EPAGRI. A Cultura da Macieira. 2 ed. Florianópolis: EPAGRI, 2002. p.743.

FULEKI, T.; FRANCIS, F. J. Quantitative determination of anthocyanins. Determination of total anthocyanins and degradation index for cranberry juice. Journal Food Science, v. 33, n. 1, p. 78-83, 1968.

GIL, M. I.; HOLCROFT, D. M.; KADER, A. A. Changes in strawberry anthocyanins and other polyphenols in response to carbon dioxide treatments. Journal Agricultural of Food Chemistry, v. 45, n. 5, p. 1662-1667, 1997.

KAUR, C.; KAPOOR, H. C. Antioxidants in fruits and vegetables - the millennium's health. International Journal of Food Science and Technology, v. 36, n. 7, p. 703-725, 2001.

LATTANZIO, V. et al. Low temperature metabolism of apple phenolics and quiescence of Phlyctaena vagabunda. Journal of Agricultural and Food Chemistry, v. 49, n. 12, p. 5817-5821, 2001.

LEA, A.; DRILLEAU, J. F. Cider-making. In: Fermented Beverage Production. London: Ed. LEA, 2003. p. 59-87.

LEE, K. W. et al. Major phenolics in apple and their contribution to the total antioxidant capacity. Journal Agricultural of Food Chemistry, v. 51, n. 22, p. 6516-6520, 2003.

MATHEW, A. G.; PARPIA, H. A. B. Food browning as a polyphenol reaction. Advance Food Research, v. 75, n. 19, p. 75-145, 1971.

MAZZA, G.; MINIATI, E. Anthocyanins in fruits, vegetables, and grains. Boca Raton, FL: CRC Press, Inc., 1994. 362p.

McGHIE, T. K.; HUNT, M.; BARNETT, L. E. Cultivar and growing region determine the antioxidant polyphenolic concentration and composition of apples grown in New Zealand. Journal of Agricultural and Food Chemistry, v. 53, n. 8, p. 3065-3070, 2005.

NICOLAS, J. J. et al. Enzymatic browning reactions in apple and apple products. Critical Reviews in Food Science and Nutrition, v. 34, n. 2, p. 109-157, 1994.

NOGUEIRA, A. Tecnologia de processamento sidricola. Efeitos do oxigênio e do nitrogênio na fermentação lenta da sidra. Curitiba, 2003. PhD Thesis, Universidade Federal do Paraná.

OOMAH, B. D.; MAZZA, G. Functional Foods. In: FRANCIS, F. J. The Wiley Encyclopedia of Science \& Technology. 2 ed. New York, NY: Wiley, 2000. v. 2, p. 1176-1182.
OSZMIANSKI, J.; LEE, C. Y. Enzymic oxidative reaction of catechin and chlorogenic acid in a model system. Journal of Agricultural and Food Chemistry, v. 38, n. 5, p. 1202-1204, 1990.

PEREZ-ILZARBE, J.; HERNANDEZ, T.; ESTRELLA, I. Phenolic Compounds in Apples: varietal differences. Zeitschrift für Lebensmittel - Untersuchung und Forschung, n. 6, p. 551-554, 1991.

PODSEDEK, A. et al. Compositional characterisation of some apple varieties. European Food Research and Technology, v. 210, n. 4, p. 268-272, 2000.

PULIDO, R.; BRAVO, L.; SAURA-CALIXTO, F. Antioxidant activity of dietary polyphenols as determined by a modified ferric reducing/ antioxidant power assay. Journal of Agricultural and Food Chemistry, v. 48, n. 8, p. 3396-3402, 2000.

REAY, P. F. The role of low temperatures in the development of the red blush on apple fruit ("Granny Smith"). Science Horticulturae, v. 79, n. 1-2, p. 113, 1999.

RICE-EVANS, C.; MILLER, N.; PAGANGA, G. Antioxidant properties of phenolic compounds. Trends in Plant Science, v. 2, n. 4, p. 304-309, 1997.

SHAHIDI, F.; NACZK, M. Phenolics in Food and Nutraceticals. New York: CRC Press, 2004. 365p.

SHAHIDI, F.; HO, C. T. Phytochemicals and phytopharmaceuticals. Champaign: ACS Press, 2000.

SHAHIDI, F.; FINLEY, J. W. Omega-3 fatty acids: Chemistry, nutrition and health effects. Washington: American Chemistry Society, 2001. ACS Symposium Series 788.

SHI, J.; MAZZA, G.; MAGUER, M. L. Functional foods: Biochemical and Processing Aspects. Ontaryo: CRC Press, 2002. p. 432.

SINGLETON, V. L.; ROSSI, J. A. Colorimetry of total phenolic with phosphomolibdic acid reagent. American Journal of Enology and Viticulture, n. 16, p. 144-158, 1965.

SPANOS, G. A.; WROLSTAD, R. E. Phenolics of apple, pear, and white grape juices and their changes with processing and storage-a review. Journal of Agricultural and Food Chemistry, v. 40, n. 9, p. 1478-1487, 1992.

TSAO, R. et al. Which polyphenolic compounds contribute to the total antioxidant activities of apple? Journal of Agricultural and Food Chemistry, v. 53, n. 12, p. 4989-4995, 2005.

Van DER SLUIS, A. A. et al. An improved, rapid in vitro method to measure antioxidant activity. Application on selected flavonoids and apple juice. Journal of Agricultural and Food Chemistry, v. 48, n. 9, p. 4116-4122, 2000.

Van DER SLUIS, A. A. et al. F. Activity and concentration of polyphenolic antioxidants in apple: Effect of cultivar, harvest year, and storage conditions. Journal of Agricultural and Food Chemistry, v. 49, n. 8, p. 3606-3613, 2001.

WROLSTAD, R. E. Anthocyanins, in Natural Food Colorants: Science and Technology. Lauro, G. J.; Francis, F. J. (Eds.). New York: Marcel Dekker Inc., 2000. p. 237-252.

YANISHLIEVA-MASLAROVA, N. V.; HEINONEN, I. M. Sources of natural antioxidants: vegetables, fruits, herbs, spices and teas. In: POKORNY, J.; NEDYALKA YANISHLIEVA, M. G. Antioxidants in Foods. Boca Raton: Academic Press, 2001.

ZABETAKIS, I.; LECLERC, D.; KAJDA, P. The effect of high hydrostatic pressure on strawberry anthocyanins. Journal of Agricultural and Food Chemistry, v. 48, n. 7, p. 2749-2754, 2000. 